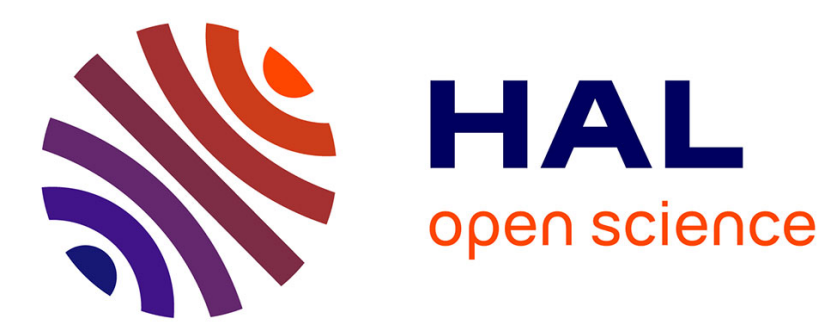

\title{
LANDAU THEORY AND MARTENSITIC PHASE TRANSITIONS
}

\author{
F. Falk
}

\section{To cite this version:}

F. Falk. LANDAU THEORY AND MARTENSITIC PHASE TRANSITIONS. Journal de Physique Colloques, 1982, 43 (C4), pp.C4-3-C4-15. 10.1051/jphyscol:1982401 . jpa-00221945

\section{HAL Id: jpa-00221945 https://hal.science/jpa-00221945}

Submitted on 1 Jan 1982

HAL is a multi-disciplinary open access archive for the deposit and dissemination of scientific research documents, whether they are published or not. The documents may come from teaching and research institutions in France or abroad, or from public or private research centers.
L'archive ouverte pluridisciplinaire HAL, est destinée au dépôt et à la diffusion de documents scientifiques de niveau recherche, publiés ou non, émanant des établissements d'enseignement et de recherche français ou étrangers, des laboratoires publics ou privés. 
JOURNAL DE PHYSIQUE

ColZoque C4, supplément au $n^{\circ} 12$, Tome 43, décembre 1982

page $\mathrm{C} 4-3$

\title{
LANDAU THEORY AND MARTENSITIC PHASE TRANSITIONS
}

\author{
F. Falk \\ Universität-GH-Paderborm, FB 6, D-479 Paderborn, F.R.G.
}

(Accepted 9 August 1982)

\begin{abstract}
Landau theory proved itself appropriate for describing phase transitions in systems such as ferroelectrics and ferromagnets. Primarily Landau es tablished the theory for second order phase transitions. Later on Devonshire generalized Landau's approach to first order transitions. The essential point of Landau theory is a power series expansion of the free energy, depending on temperature and on an order parameter describing the phase transition. In order to deal with phase boundaries the theory has been generalized to Ginzburg-Landau theory by adding a term depending on the gradient of the order parameter. Inspite of the success of Landau and GinzburgLandau theory in the systems mentioned above only little work has been done concerning martensitic phase transitions. Difficulties arise from the fact that the deformation of the unit cell does not coincide with the macroscopic strain. Considerations for overcoming this problem are discussed. It seems that even in the case of martensitic phase transitions Landau theory may be used as a starting point to obtain deeper insight into phenomena such as soft modes, nucleation, and the role of Tattice defects.
\end{abstract}

1. Introduction. - For more than five decades martensitic phase transitions have been studied with growing effort. In the beginning the notion of martensite was defined for ferreous alloys, namely stee 1 . Later on phase transitions very similar to the classical martensitic one were discovered in a lot of non-ferrous alloys too. Today it seems that the transitions in non-ferreous alloys are more typical and show the underlying principles more clearly. From the very beginning martensitic phase transitions were the domain of metallurgists. Inspite of the high practical significance in materials science and technology, physicists looked upon this type of phase transitions in steels as dirty and unpromising. Instead phase transitions such as liquid-vapour or ferromagnetic-paramagnetic were in vogue. Later the evolution in both the areas took place rather independently.

In physics phase transitions were dealt with, in the beginning, by phenomenological theories which simply describe the phenomena on a macroscopic scale containing many atoms. The first equation of state representing a phase transition was suggested by van der Waals in 1873. In 1937 Landau established his famous theory of second order phase transitions [1] which was extended by Devonshire $[2,3]$ to first order phase transitions in 1949. In order to deal with phase boundaries the phenomenological theories were generalized to Ginzburg-Landau theory. Later on the microscopic foundation of the theories mentioned above was pushed forward. From statistical mechanics the phenomenological theories can be drived using the mean field approximation neglecting fluctuations. In spite of this restriction the phenomenological theories are very successful in describing a lot of phase transition phenomena. Nevertheless they were not applied to martensitic phase transitions until recently, presumably because of a lack in collaboration between metallurgy and physics.

The aim of this paper is to give a survey both, of the phenomenological theories of phase transitions and of their application to martensitic phase transitions. In Chapter 2 we present the basic ideas of the phenomenological theories (Landau, Devonshire, and Ginzburg-Landau theory). In Chapter 3 applications are discussed. The last chapter deals with martensitic transitions and their description using 
the theories mentioned above. The specific problems as well as the possible scope are outlined.

\section{Phenomenological theories of phase transitions}

\subsection{Basic ideas}

A phenomenological theory for a specific system is established in two steps.

The first step is to find a quantity, the order parameter e, characterizing the phase transition that is the difference of both the phases. This problem is not a trivial one requiring a deep insight into the particular phase transition. The order parameter is an internal variable of the system. Examples can be found in Table 1. In the beginning the notion "order parameter" was used for the magnetization in the paramagnetic-ferromagnetic phase transition where, in the paramagnetic phase, the spins are disordered whereas in the ferromagnetic phase the spins are parallet. Later on the notion was used even in phase transitions not connected with orderdisorder phenomena. In martensitic phase transitions the order parameter is the strain (see Chapter 4 ).

In the second step we determine the equations of state by constructing a free energy $f$ as a function of temperature $T$ and order parameter e. From $f(e, T)$ every thermodynamic quantity can be calculated. Especially the order of the phase transsition is fixed. It must be emphasized that one single free energy function represents both the phases.

Let us discuss the equilibrium. Without an external influence the equilibrium of an isothermal system is given by the minimum of the free energy $f$ with respect to the order parameter. This condition yields $\partial f(e, T) / \partial e=0$. For convenience one defines the quantity

$$
\sigma=\partial f(e, T) / \partial e
$$

describing the energetic response of the system due to a changing internal state. In equilibrium the order parameter adjusts in such a way that $\sigma$ vanishes. In the martensitic case $\sigma$ is the stress. If there is an external influence the situation

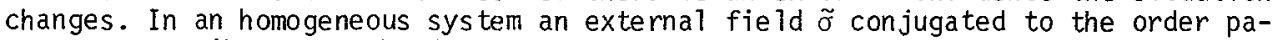
rameter contributes to the free energy by -õe. The physical significance of $\tilde{\sigma}$ has to be determined for each particular phase transition (for examples see Table 1). In this case the total free energy is given by

$$
f_{\tilde{\sigma}}(e, T)=f(e, T)-\tilde{\sigma} e .
$$

As a consequence the equilibrium shifts, under the influence of an external field, to the minimum of $f_{\tilde{\sigma}}$. This condition yields

$$
\sigma(e, T)=\tilde{\sigma}
$$

which means that the external field is balanced by the internal response quantity. Eq. 2 determines the equilibrium value of the order parameter as a function of the external field and temperature.

\subsection{Landau theory}

Landau in his theory of second order phase transitions assumed the free energy to be an analytic function of the order parameter and of temperature. Therefore he expanded the free energy density function $f$, with respect to the order parameter $e$, into a power series

$$
f(e, T)=f_{0}+A e+B e^{2}+C e^{3}+D e^{4}+\ldots
$$

where the coefficients are analytic functions of the temperature T. If e, as usual, is chosen in such a way that the high temperature phase without external field corresponds to vanishing $e$, then one must have a minimum of $f$ at $e=0$ for $T>T_{c}$ and consequently $A=0$. In order to get a second order phase transition at $T_{C}$ the ${ }^{C}$ minimum of $f$ must shift continuously for $T<T_{C}$. Therefore $B$ must change its sign at $T_{C}$ with $B>0$ for $T>T_{C}$. The simplest possibility to get this behaviour is

$$
B=b\left(T-T_{C}\right) \quad 0<b=\text { const. }
$$


Furthermore one must have $C=0$ and $D>0$. Landau assumed $D$ to be constant. Consequently the most simple free energy function showing a second order phase transition is obtained (Fig. la)

$$
\begin{aligned}
f(e, T)= & f_{0}(T)+b\left(T-T_{C}\right) e^{2}+D e^{4} \\
& b, D, T_{C}>0, \text { const. }
\end{aligned}
$$

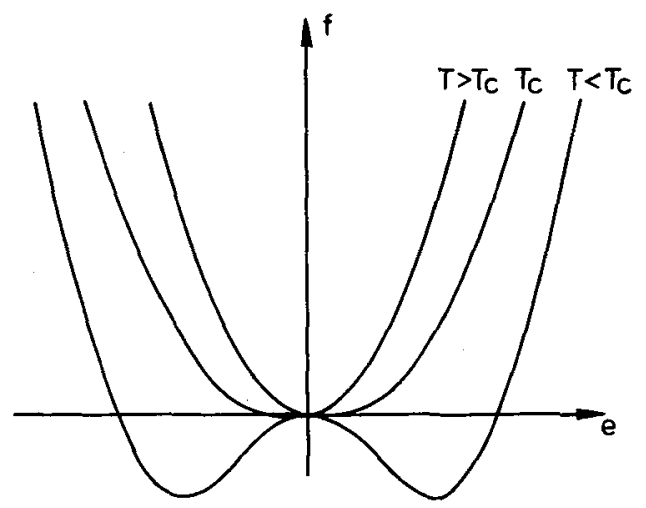

Fig. Ia Landau free energy (Eq. 3) as a function of order parameter.

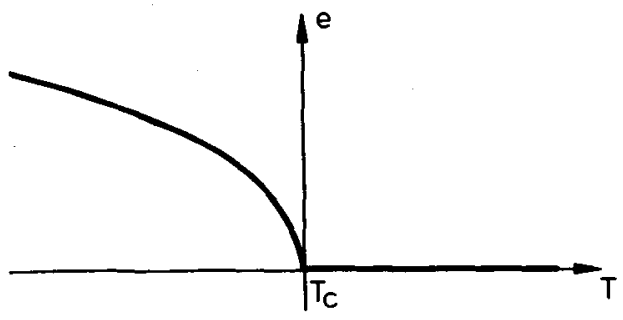

Fig. 1b Equilibrium order parameter (Landau theory) as a function of temperature if there is no external field. Second order phase transition at $T_{C}$.

Let us discuss the consequences of Eq. 3 in the case of vanishing external field. Fig. Ib shows the equilibrium value of the order parameter as a function of temperature following from $\mathrm{Eq}$. 2. At high temperatures $\left(T>T_{C}\right)$ the order parameter vanishes. If the temperature is lowered beyond $T_{C}$ the order parameter changes continuously. However the derivative of e with respect to temperature jumps at $T_{c}$ indicating a second order phase transition. Two possibilities have to be distinguished. Following physical arguments it may be that the order parameter cannot be negative. Then there is oniy one minimum of $f$ at each temperature. However, if the order parameter may take positive or negative values then there are, at low temperatures $\left(T<T_{C}\right)$, two symmetric minima of the free energy. Since the absolute value of the order parameter is the same for both the minima, the corresponding phases are identical differing only in their orientation. Which orientation is present cannot be predicted. It may happen that the system splits into domains of different orientation.

If there is an external field the situation changes considerably. (Fig. 2a). To begin with, consider the case where the external field is fixed whereas temperature is changed. From Eqs. 2, 3 one obtains in equilibrium an e-T curve plotted in Fig. $2 b$. It is noticed that there is no phase transition at all. Under the influence of the external field the order parameter differs from zero even at high temperature. on cooling e gradually increases to approach the curve of vanishing field asymptotical Ty.

Let us now discuss the case of fixed temperature and varying external fjeld. By minimizing $f_{\tilde{\sigma}}$ (Eqs. 2, 3, Fig. 3a) one gets the following cubic equation for the equi librium order parameter as a function of temperature and external field (Fig. 3b)

$$
\tilde{\sigma}=2 b\left(T-T_{C}\right) e+4 D e^{3}
$$




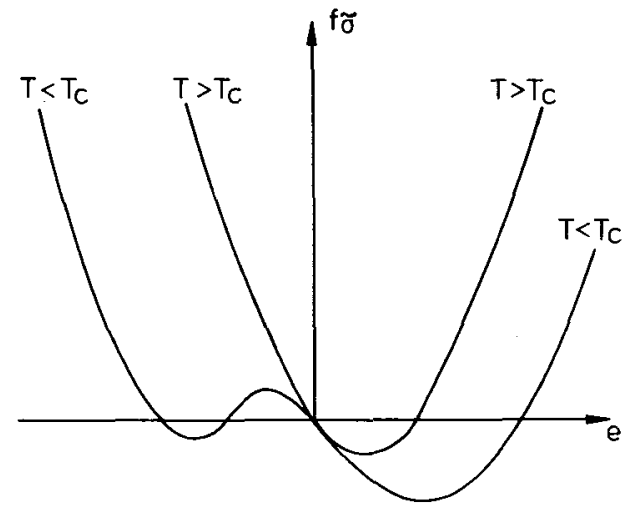

Fig. 2a Landau free energy under the influence of an external field $\tilde{\sigma}>0$ as a function of the order parameter.

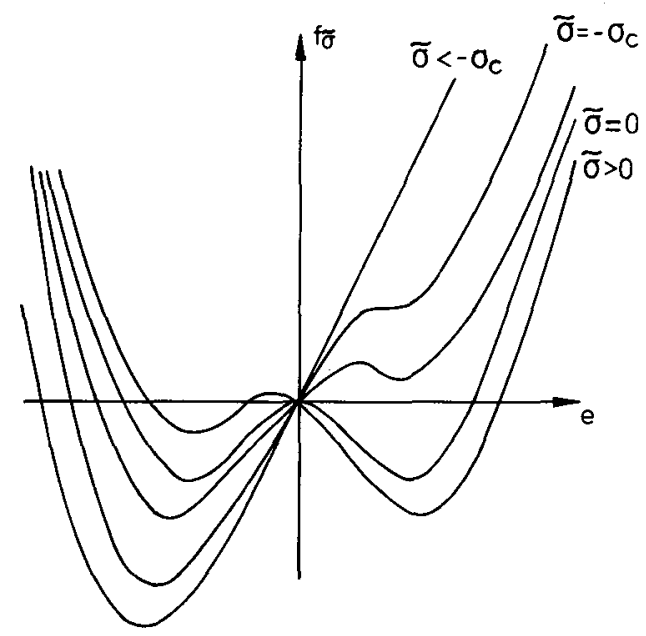

Fig. 3a Landau free energy as a function of order parameter for different values of the external field in the case $T<\mathrm{T}_{C}$

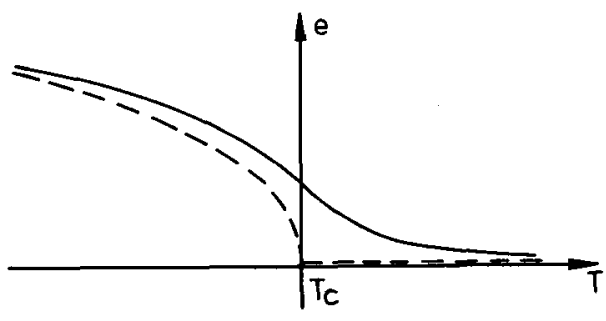

Fig. 2b Equilibrium order parameter (Landau theory) as a function of temperature under the influence of an external field $\tilde{\sigma}>0$. The dashed curve corresponds to vanishing external field.

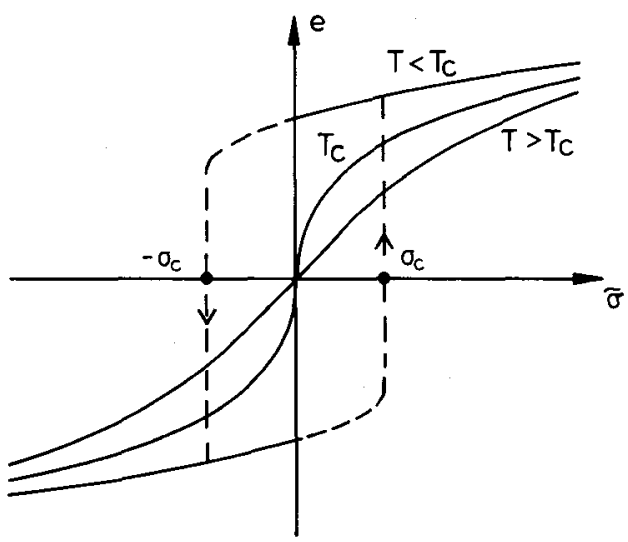

Fig. 3b Equilibrium value of the order parameter (Landau theory) as a function of the external field for different temperatures. First order phase transition for $T<T_{C}$. 
At high temperatures $\left(T>T_{C}\right)$ the order parameter changes continuously with $\tilde{\sigma}$. However, for $T<T_{C}$ a first order phase transition results. If the external field is diminished the order parameter decreases too. Even at vanishing field there is a "remanence". Applying an external field in the opposite direction, the right minimum of $f \tilde{\sigma}$ vanishes only at $-\sigma_{C}(T)$. With th is field the phase becomes unstable and therefore the system jumps in to the left minimum of $f_{\tilde{\sigma}}$. In the range $0>\tilde{\sigma}>-\sigma_{C}$ the right minimum is lying at higher energy than the left one. Therefore the right phase is only metastable. The equilibrium phase transition occurs at $\tilde{\sigma}=0$ where the minima are of equal depth. If the field is reversed, the retransformation must occur at least at $\sigma_{c}$. Therefore, one may get hysteres is in the field induced phase transition. The maximum value of the hysteres is is given by $\sigma_{c}$. However, Landau theory is unable to predict the extent to which hysteresis does occur in reality.

The most important properties of phase transitions described by Eq. 3 are

1. The temperature-induced phase transition is of second order if the external field vanishes. There is no hysteresis.

2. There is no temperature-induced phase transition if an external field is applied.

3. The field-induced phase transition at $T<T_{C}$ is of first order and is associated with hysteresis.

4. There is no field-induced phase transition at $T>T_{C}$.

\subsection{Devonshire theory}

Landau theory of second order phase transition has been successfully applied to various systems such as ferro magnetic and ferroelectric materials. However, there are other materials, such as the ferroelectric. $\mathrm{BaTi}_{3}$, which exhibit a temperatureinduced first order phase transition combined with hysteresis that is not covered by Eq. (3). Devonshire [2,3] has estab7ished a thermodynamic theory for this kind of phase transitions. To this end he looked for a free energy function complying with the following requirements. At high temperatures the free energy should have one minimum only at vanishing order parameter representing the high temperature phase. At low temperatures, where only the second phase is stable, the free energy should have a minimum at non-vanishing order parameter. At intermediate temperatures, the free energy must have minima corresponding to both the phases. With the additional assumption that the free energy should be an even function of the order parameter Devonshire $[2,3]$ ended up with the following ansatz (Fig. 4)

$$
\begin{aligned}
& f(e, T)=f_{0}(T)+A(T) e^{2}-B e^{4}+C e^{6} \\
& A(T)=a\left(T-T_{1}\right) \quad a, B, C, T_{1}>0 \text {, const. }
\end{aligned}
$$

It should again be emphasized that one free energy function applies to both the phases. It may be noted that the minima corresponding to the low-temperature phase do not continuously evolve from the high-temperature. phase. On the contrary they suddenly appear at

$$
T_{2}=T_{1}+\frac{1}{3} \frac{B^{2}}{a C}>T_{1} \text {. }
$$

At $T_{0}$ with

$$
T_{2}>T_{0}=T_{1}+\frac{1}{4} \frac{B^{2}}{a C}>T_{1}
$$

the minima are of equal depth. In the range $T_{2}>T>T_{0}$ the low-temperature phase is metastable, whereas in the range $T_{0}>T>T_{1}$ the high-temperature phase is metastable. At $T_{1}$ the $h$ igh-temperature phase becomes unstable. Because of symmetry the low-temperature phase exists in two orientations. The response of the system to a changing order parameter is given by

$$
\sigma(e, T)=\partial f / \partial e=2 A(T) e-4 B e^{3}+6 C e^{5}
$$




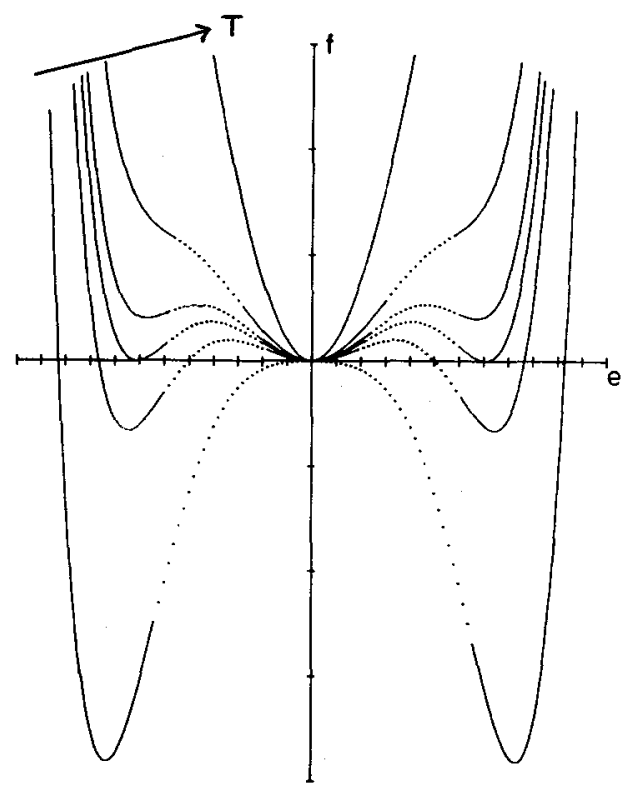

Fig. 4 Devonshire free energy as a function of order parameter for different temperatures (Eq. 5)

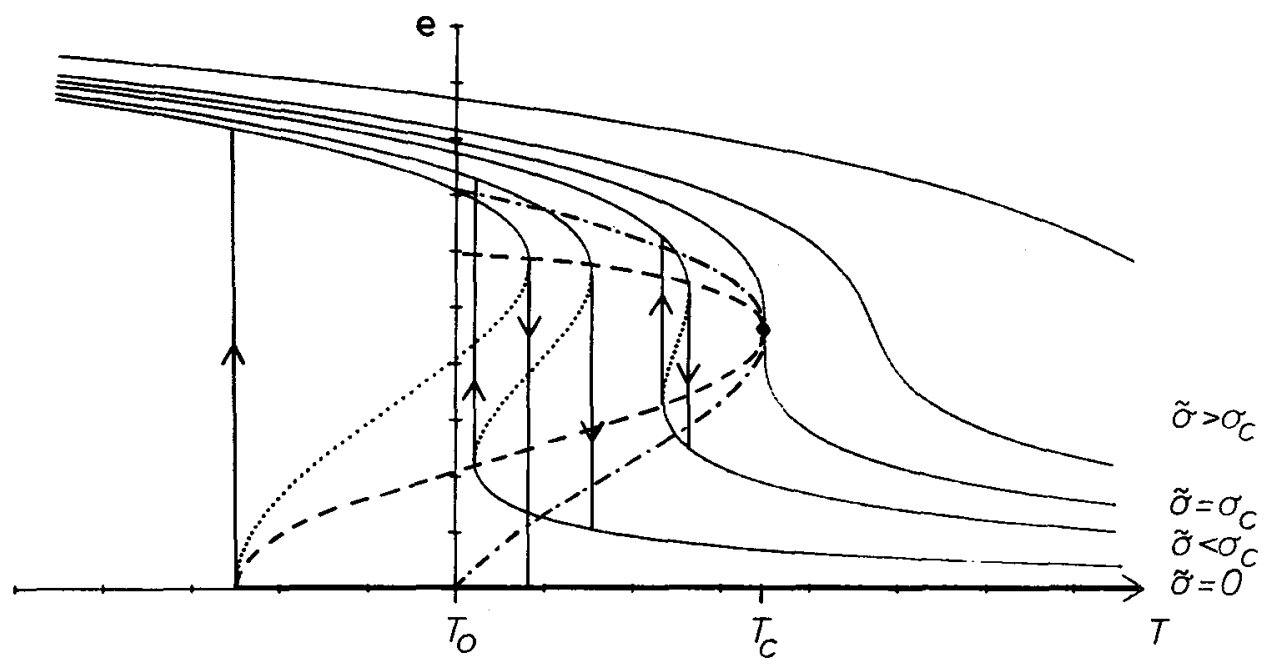

Fig. 5 Equilibrium order parameter (Devonshire theory) as a function of temperature for different values of the external field $\tilde{\sigma}$. First order phase transition for $\tilde{\sigma}<\sigma_{c}$. The dotted part of the curves bounded by the spinodal (dashed curve) corresponds to unstable states decomposing into two phases. The dashdotted curve represents the locus of the equilibrium phase transition. critical point at $T_{C}, \sigma_{C}$. 
In equitibrium $\sigma$ has to be balanced by an external field $\tilde{\sigma}$ (Eq. 2) yielding the equilibrium value of the order parameter as a function of $T$ and $\widetilde{\sigma}$.

To begin with the case of fixed external field and varying temperature (Fig. 5) may be discussed. Above

$$
\sigma_{c}=\frac{16}{25 \sqrt{5}} \sqrt{\frac{B^{5}}{c^{3}}}
$$

there is no temperature-induced phase transition. Changing temperature we gradually alter the order parameter. At ${ }^{\circ} \mathrm{C}, \mathrm{T}_{\mathrm{C}}$, $\mathrm{e}_{\mathrm{C}}$

$$
T_{c}=T_{1}+\frac{3}{5} \frac{B^{2}}{a C} \quad e_{c}=\sqrt{\frac{B}{5 C}}
$$

there is a critical point. Below $\sigma_{c}$ one gets a temperature-induced first order phase transition with hysteresis. The points on the dotted part of the e-T-curves bounded by the dashed curve (spinodal, Fig. 5) obey the equilibrium condition (Eq. 2). However, they correspond to a maximum of the free energy. Therefore, they are unstable points. For given values of the external field, the order parameter, and temperature lying in this domain the system decomposes into two phases. To get the order parameter of both the phases only the e-values which correspond to the prescribed temperature on the solid branches of the e-T curve need be considered. Suppose, the hightemperature phase is cooled in a fixed external field below $\sigma_{c}$. At the temperature where the e-T-curve meets the dash-dotted curve the free energy of both the phases is the same. Therefore, this point defines the equilibrium phase transition that is the foremost possibility of the phase transition at all. On further cooling, the high temperature phase becomes metastable getting unstable only at the beginning of the dashed curve where the phase transition must occur at the latest (left arrow in Fig. 5). The domain between the dashed and dash-dotted curve corresponds to metastability. On heating the low-temperature phase retransforms at a temperature between the equilibrium phase transition point and the right arrow (Fig. 5) where this phase becomes unstable. As a consequence there is a hysteresis. Phenomenological theories are not capable of predicting the extent to which hysteresis does occur in a real system. They only give an upper limit.

At the critical point $\left(T_{C}, \sigma_{C}\right)$ the region of coexisting phases ends. Therefore the high temperature phase can be gradually converted into the low-temperature phase without any phase transition by surrounding the critical point.

In addition to the temperature-induced phase transition there is a field-induced phase transition. In Fig. 6 б-e-curves are plotted for different temperatures. The interpretation of the curves in Fig. 5 and 6 is based on the same considerations. Below $T_{1}$ only the low temperature phase is stable. There is a first order fieldinduced phase transition between both the orientations of this phase. In the range $T_{2}>T>T_{1}$ in addition to this type of transition there is a phase transition from the high-temperature phase (large e) to the low-temperature phase (sma $11 \mathrm{e}$ ). In the domain $T_{c}>T>T_{2}$ this latter transition is the only possible one, whereas above $T_{C}$ a field-induced phase transition cannot occur. It may be noticed that the stressstrain curves of shape-memory alloys look very similar to the $\sigma$-e curves in Fig. 6. Therefore it may be supposed that Devonshire theory is appropriate for describing martensitic phase transitions with the strain as order parameter. This is further discussed in the last chapter.

The most important results of Devonshire theory thus are:

1. First order temperature-induced phase transition with hysteresis be low $\sigma_{c}$.

2. First order field-induced phase transition between low and high temperature phases in the range $T_{C}>T>T_{2}$.

3. First order field-induced phase transition between different orientations of the low temperature phase in the range $T<T_{1}$.

4. Existence of a critical point. 

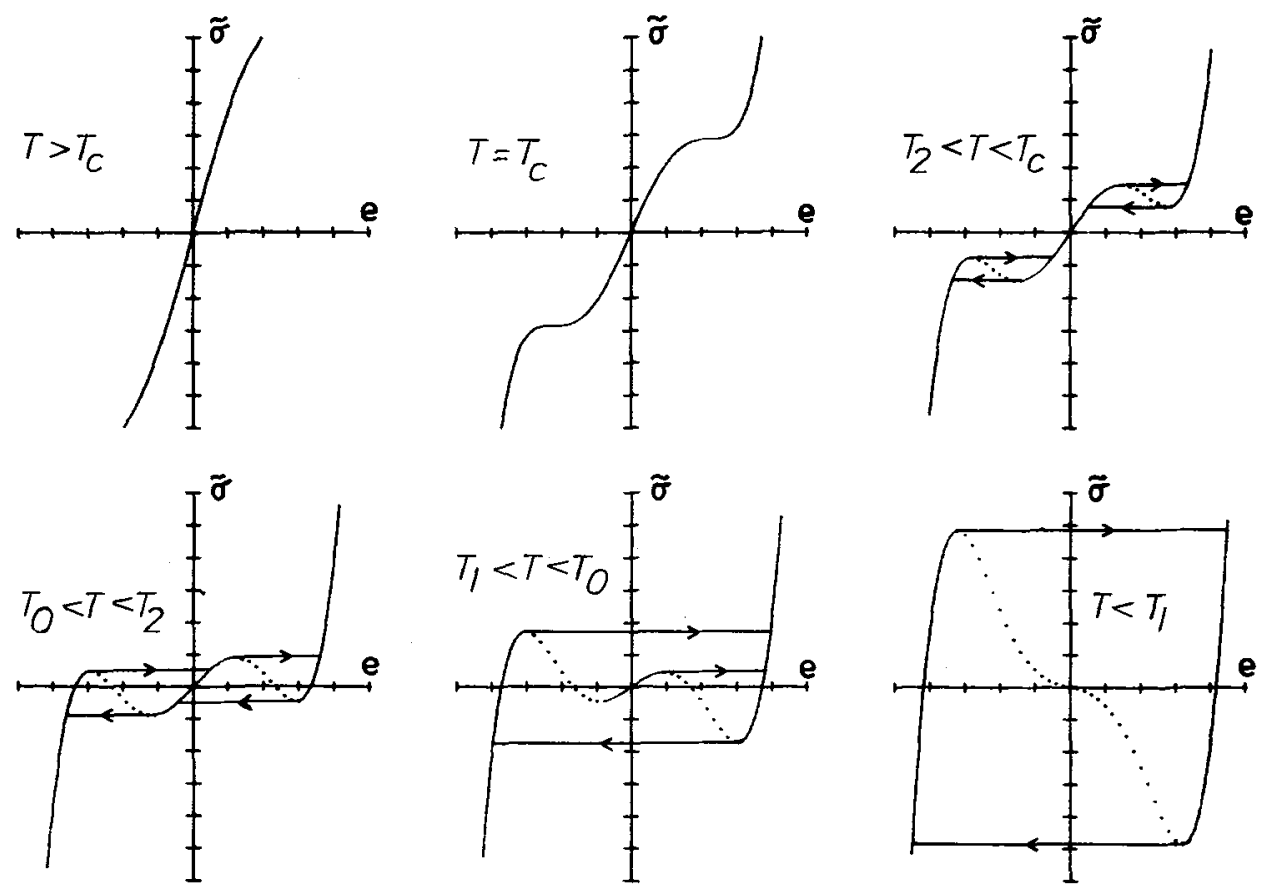

Fig. 6 Equilibrium order parameter (Devonshire theory) as a function of external field for different temperatures. The dotted part of the curves corresponds to unstable states decomposing into different phases.

2.4 Ginzburg-Landau theory. - There are first order phase transitions in Landau as welT as in Devonshire theory. Connected with these first order phase transitions is the existence of phase equilibrium. This means that at certain values of temperature and external field different phases or different orientations of the same phase are in equilibrium and may coexist in the same body showing a domain structure. At the domain boundaries the order parameter changes rapidly. If this situation was treated using Landau or Devonshire theory one would get domain walls of vanishing width and vanishing energy which obviously is not correct. The reason for this result is that a free energy depending on the order parameter is appropriate for situations only where this quantity varies slowly. Dealing with domain boundaries the free energy density must be modified by terms containing derivatives of the order parameter. It appears that a gradient term is sufficient. However, this assumption can be confirmed by its success rather than by first princip.les. It may be concluded from symmetry arguments thatan order parameter gradient cannot occur linearly. The lowest possible term is a quadratic one. For an isotropic system, the Ginzburg-Landau free energy reads

$$
f(e, \nabla e, T)=f_{H}(e, T)+\alpha(\nabla e)^{2}
$$

where $f_{H}$ is the Landau or Devonshire free energy (Eqs. 2, 5, H stands for homogeneous). $\alpha$ has to be positive. Otherwise the system could lower its energy indefinitely by creating an infinite number of domains. Usually it is sufficient to assume $\alpha$ to be a positive constant (for particular systems, the references are cited in the following chapter). The equilibrium of an isothermal system is given, in the absence of an external field, by the minimum of 


$$
F_{\text {tot }}=\int f(e(x), \nabla e(x), T) d V
$$

Since this condition determines the order parameter as a function of the position $x$, the structure of domain walls follows. Especially their width is obtained. Inserting the equilibrium structure $e(x)$ into Eq. 8 results in the energy of a domain wall.

3. Application to specific systems. - The paramagnetic-ferromagnetic phase transition is a typical problem for Landau theory. The order parameter and the corresponding external field are the magnetization vector and the external magnetic field, respectively. If both vectors are lying in one fixed direction they may be looked upon as scalars. In this case the observed behaviour of ferromagnetic system is described quite well by Eq. 3. The corresponding Landau free energy can be derived from a microscopic Ising model using the mean-field approximation.

If the magnetization is not restricted to one direction then the situation is more complicated [5]. Since there is an interaction between magnetism and the crystal lattice (magnetostriction) the free energy must be modified by appropriate terms. Additionally, the energy of the stray field must be included. On the basis of a modified theory of Ginzburg-Landau type (micromagnetic equations) walls separating differently oriented ferromagnetic domains have been treated [6]. Depending on the dimension of the body different types of walls (Bloch or Neél walls) are obtained. In addition, interaction phenomena between walls and crystal lattice defects have been treated. The phenomenological theory proved itself as a very useful tool in dealing with problems of ferromagnetic bodies.

Another type of magnetic phase transition is the antiferromagnetic-paramagnetic one. In the antiferromagnetic phase magnetic moments of neighbouring atoms located on different sublattices are antiparalle 1 . Therefore, there is no net magnetization. The order parameter is the difference in the magnetization of both the sublattices. The response is the "staggered field" which has no realization as an external field. Therefore, antiferromagnetism is a typical example of a phase transition without corresponding external field.

Devonshire $[2,3]$ dealt with ferroelectric materials with the polarization as order parameter. The response corresponding to an external field is the electric field. According to the material second order or first order phase transitions are observed. A typical example where Landau theory (Eq. 3) has been used is Triglycine Sulfate whereas $\mathrm{BaTiO}_{3}$ has been described by Devonshire theory (Eq. 5). Since ferroelectric materials show electrostriction, an interaction energy containing lattice deformation must be added. Details can be found in the book by Grindlay [7]. Basing on a Ginzburg-Landau theory domain walls have been studied by Mitsui and Furuchi [8], Ivanchik [9] and BuTaevski i [10].

In Table 1 various types of phase transitions which have been treated using Landau, Devonshire, or Ginzburg-Landau theory are listed. A survey of Landau theory and of its foundation by means of statistical mechanics can be found in [11].

4. Martensitic phase transitions. - Martensitic phase transitions are defined as diffusionless solid state structural phase transitions of first order with a deformation of the lattice such that a macroscopic strain results [12]. Occasionally the first order condition is not included in the definition. The following concerns alloys that exhibit pseudoelasticity, ferroelasticity, and shape memory effect as a consequence of the martensitic phase transition (for a review on these effects, refer [13]).

Attempts have been made to describe martensitic phase transitions by means of phenomenological theories. A Landau-type description of the A15 martensitic phase transition has been given by Bhatt [14]. The order parameter is the energy level of electronic bands. The transformation strain results from a coupling term between order parameter and strain. However, the microscopic background in the A15 transformation seems to be quite different from these in the systems considered here. For cubic-tetragonal phase transformations Anderson and Blount [15], Axe and Yamada [16], and Sakhnenko and Talanov [17] have presented a Landau description using the strain as order parameter. Since this quantity is a tensor of the second rank, group theoretical arguments have been used to find appropriate combinations of the strain com- 
TA B L E 1

\begin{tabular}{|c|c|c|c|c|}
\hline $\begin{array}{c}\text { phase } \\
\text { transition }\end{array}$ & $\begin{array}{l}\text { order } \\
\text { parameter }\end{array}$ & $\begin{array}{l}\text { external } \\
\text { field }\end{array}$ & $\begin{array}{l}\text { type of } \\
\text { theory* }\end{array}$ & reference \\
\hline ferromagnetic & magnetization & magn.field & $\begin{array}{l}\mathrm{L} \\
\mathrm{M} \\
\mathrm{G}\end{array}$ & $\begin{array}{l}44 \\
6,35 \\
6\end{array}$ \\
\hline antiferromagne tic & $\begin{array}{l}\text { difference of } \\
\text { magnetizations }\end{array}$ & none & G & 6,41 \\
\hline ferroelectric & polarization & electr.field & $\begin{array}{l}\text { L } \\
D \\
G\end{array}$ & $\begin{array}{l}2,3 \\
2,3 \\
8,9,10\end{array}$ \\
\hline Tiquid-vapour & density & pressure & $\begin{array}{l}M \\
G\end{array}$ & $\begin{array}{l}4 \\
36,37,38\end{array}$ \\
\hline superconductive & $\begin{array}{l}\text { density of Cooper- } \\
\text { pairs }\end{array}$ & none & G & 39,40 \\
\hline structural & $\begin{array}{l}\text { displacement, } \\
\text { rotation }\end{array}$ & none & $L, D$ & 35 \\
\hline order-disorder & long range order & none & $\begin{array}{l}L \\
G\end{array}$ & $\begin{array}{l}42 \\
43\end{array}$ \\
\hline martensitic & strain & stress & $\begin{array}{l}\text { see } \\
\text { Chap. } 4\end{array}$ & \\
\hline
\end{tabular}

*L Landau theory, D Devonshire theory, M Modified Landau or Devonshires theory, G Ginzburg-Landau theory.

ponents obeying the required symmetry. Recently Kelly and Stobbs $[18,19]$ have proposed a Landau theory for B-phase alloys with the amplitude of charge density waves serving as order parameter. They have introduced a coupling to phonon amplitudes as well as to strain. However, there are no results concerning the thermodynamic consequences of the model.

Whereas the above quoted papers start from a microscopic background, Ericksen [20] has proceeded from theory of thermoelasticity. However, his paper is concerned with continuous (second order) martensitic transformation which is not a realistic simplification. Parry [21] starting from nonlinear theory of elasticity has developed a rather general theory of thermoelastic phase transitions. While attention is given in his paper to universal properties of thermodynamic potentials describing structural phase transitions and twinning, it does not, unfortunately, provide an application to martensitic phase transition.

Looking at observed stress-strain curves of shape-memory alloys [13] a more direct approach appears quite obvious. A striking similarity to the curves in Fig. 6 is noticed, showing the response quantity $\sigma$ as a function of the order parameter $e$ for various temperatures as derived from Devonshire theory (Eqs. 5, 6). This observation has prompted the present author $[22,23,24]$ to establish a one-dimensional model of martensitic phase transitions where the shear strain and the shear stress were identified with the order parameter and response, respectively. Devonshire theory (Eq. 5) yields not only the observed stress-strain curves (ferroelasticity, pseudoelasticity) but also shape-memory effect, lattice softening and so on. The author has developed, based on the one-dimensional model, a Ginzburg-Landau theory which allows for treating static and moving domain walls between martensite variants as well as between austenite and martensite [25]. The discrete version of the one-dimensiona] model 
stems from Suzuki and Wuttig [26] who have dealt numerically with nonlinear strain waves which steepen to from martensitic nuclei. Generalizing the model to three dimensions the following two problems are met which necessarily arise in any phenomenological theory of martensitic phase transitions.

1. The combination of the components of the strain tensor to be used as order parameter.

2. The scale to be used in defining the relevant strain.

The first question can be answered by group theoretical arguments. The answer, however, depends on the symmetry of the specific materia?. One must look for combinations of the strain tensor components describing the transformation which are invariant with respect to the symmetry group of the high-temperature phase (being the larger group). Whereas a11 the possible cases for second order phase transitions have been given by Toledano [27], there are only preliminary results for first order transitions [28].

The second problem concerning the scale of the relevant strain is a more difficult one. A possibility is to use the lattice deformation (Bain strain). However, the lattice deformation usually does not coincide with the macrostrain for the following reason. In a real crystal, martensite evolves from austenite in such a way that, at least in an intermediate stage, plane martensite-austenite interfaces exist. The interfaces must be invariant planes with respect to the macrostrain, which can be obtained by twinning within martensite. This consideration led to the well-known theories of Wechsler, Liebermann and Read [29], and Bowles and Mackenzie [30] relating the macrostrain to the lattice deformation. In a Ginzburg-Landau theory with lattice deformation as order parameter, an austenite-martensite interface enforces twinning by compatibility. However, if we deal with pure martensite, how can we let know the theory that martensite must be twinned internally because of its history. A way out of this dilemma could be to use the macrostrain as order parameter. Then the lattice deformation does not enter the theory. Consequently, symmetry arguments must refer to internally twinned martensite. The second problem concerning the appropriate scale is known, in statistical mechanics, as the rather general problem of coarse graining [31]. It does not occur if the twinning already is included in the lattice strain. This, for example, is the case in the bcc $\rightarrow 9 \mathrm{R}$ transformation.

Let me end with some remarks about possible applications of Landau, Devonshire or Ginzburg-Landau theory. To begin with, such a theory is a convenient phenomenological description of the martensitic phase transition. It comprises a complete nonlinear theory of thermoelasticity replacing the linear theory of elasticity which is inadequate for shape-memory alloys. A very promising application is the nucleation problem. In both, homogeneous as well as heterogeneous nucleation, Landau or Devonshire theory could modify the present ideas [32]. In the vicinity of a nucleus stress and strain concentrations of considerable amount can be expected. In the region of elastic instability the linear theory of elasticity applied usually seems a very poor approximation. A Landau-type theory has the advantage of yielding the whole temperature dependent nonlinearity, which lowers the nucleation barrier. A similar argument applies to heterogeneous nucleation on defects. The nonlinearity as well as the huge anisotropy of the elastic response modify the stress field of dislocations. Therefore their ability in favouring martensitic embryos may be increased considerably. Nucleation along nonclassical paths including diffuse interfaces with continuously changing strain [33] seems to be important in some systems (bcc $\rightarrow 9 R$, $\mathrm{fcc} \rightarrow$ hcp, [32]). In this case a Ginzburg-Landau theory is indispensable. The discrete version of Suzuki and Wuttig [26] already has shown its value [34].

As has been demonstrated Landau, Devonshire, and Ginzburg-Landau theories are promising approaches to a lot of problems concerning martensitic transformations. Even a one-dimensional model reflects characteristic features of martensite. This result encourages the development of a complete three-dimensional version of Ginzburg-Landau theory for martensitic phase transitions. 


\section{References}

1 Landau L.D., Phys. Z. Sowjetunion 11 (1937) 26, 545 (in German). English translation in: "ColTected Papers of L.D. Landau", D. ter Haar ed., 1967, p. 193.

2 Devonshire A.F., Phi1. Mag. 40 (1949) 1040; 42 (1951) 1065.

3 Devonshire A.F., Adv. Phys. 3 (1954) 85.

4 Landau L.D., Lifshitz E.M., "Statistical Physics", Pergamon Press, 0xford 1980, Chap. XIV.

5 Tdlédano P., Clin M., Hédoux M., Ferroelectrics 35 1981) 239.

6 Hubert A., "Theorie der Domänenwände in geordneten Medien", Springer, Berlin 1974 .

7 Grindlay J., "An Introduction to the phenomenological theory of ferroelectricity", Pergamon Press, 0xford 1970.

8 Mitsui T., Furuchi J., Phys. Rev. 90 (1953) 193.

9 Ivanchik I.I., Sov. Phys. Sol. St. 3 (1962) 2705.

10 Bulaevskii L.N., Sov. Phys. Sol. St. 5 (1964) 2329.

11 Gautier F. in "Solid State Phase Transformations in Metals and Alloys", Ecole d'été d'Aussois 1978, les éditions de physique, Orsay.

12 Wasilewski R.J., Met. Tr. A 67 (1975) 1405.

13 Delaey L., Krishnan R.V., Tas H., Warlimont H., J. Mat. Sci. 9 (1974) 1521, 1536,1545 .

14 Bhatt R.N., Phys. Rev. B 17 (1978) 2947.

15 Anderson P.W., Blount E.I., Phys. Rev. Lett. 14 (1965) 217.

16 Axe J.D., Yamada Y., Phys. Rev. B 24 (1981) 2567.

17 Sakhnenko V.P., Talanov V.M., Sov. Phys. Sol. St. 21 (1979) 1401, 22 (1980) 458.

18 Kelly M.J., Stobbs W.M., Scr. met. 13 (1979) 919.

19 Kelly M.J., Stobbs W.M., Phys. Rev. Lett. 45 (1980) 992.

20 Ericksen J.L., J. therm. stresses 4 (1981) 107.

21 Parry G.P., Int. J. Solids Structures 17 (1981) 361, 18 (1982) 59.

22 Falk F., ZAMM 60 (1980) T 118.

23 Falk F., Acta met. 28 (1980), 1773.

24 Falk F., Arch. mech. in press.

25 Falk F., ICOMAT 82.

26 Suzuki T., Wuttig M., Acta met. 23 (1975) 1069.

27 Tolédano J.C.\&P., Phys. Rev. B 21 (1980) 1139. 
28 Aizu K., Phys. Rev. B 23 (1981) 1292.

29 Wechsler M.S., Liebermann D.S., Read T.A., Trans. Am. Inst. Min. Metall. Eng. 197 (1953) 1503 .

30 Bowles J.S., Mackenzie J.K., Acta met. 2 (1954) 129, 138, 224.

31 Kawasaki K., Imaeda T., Gunton J.D., in "Perspectives in Statistical Physics", H.J. Raveché ed., North-H011and, Ams terdam 1981, p. 201.

32 Olson G.B., Cohen M., Proc. Conf. Solid-Solid Transf. 1981, ICOMAT 82.

33 Roitburd A.L., Kurdjumov G., Mat. Sci. Eng. 39 (1979) 141.

34 Suzuki T., J. Phys. Soc. Jpn. 45 (1978) 860.

35 Bruce A.D., Cowley R.A., "Structural phase transitions" Taylor \& Francis, London 1981.

36 van der Waals, Z. phys. Chem. 13 (1894) 657 (in German), English translation in J. stat. phys. 20 (1979) 197.

37 Cahn J.W., Hilliard J.E., J. chem. phys. 28 (1958) 258, 30 (1959) 1121, 31, (1959) 688 .

38 Widom P., in "Perspectives in Statistical Physics", H.J. Raveché ed., NorthHolland, Ams terdam 1981, p. 273.

39 Ginzburg V.L., Nuovo Cim. 2 (1955) 1234.

40 deGennes P.G., "Superconductivity in Metals and Alloys", Benjamin, New York 1966 .

41 Bar'yakhtar V.G., Brovik A.E., Popov V.A., Stefanowskii E.P., Sov. Phys. Sol. St. 12 (1971) 2659 .

42 Ziman J.M., "Model of Disorder", Cambridge University Press, Cambridge 1979.

43 Allen S.M., Cahn J.W., Acta met. 27 (1979) 1085.

44 Landau L.D., Lifshitz E.M., "Electrodynamics of Continuous Media", Pergamon, Oxford 1960, Chap. V. 